\title{
Transações com Partes Relacionadas em empresas com estrutura piramidal: uma comparação dos fatores explicativos nas controladoras e coligadas
}

\author{
Silvia Amélia Mendonça Flores \\ https://orcid.org/0000-0002-0424-8375 \\ Igor Bernardi Sonza \\ https://orcid.org/0000-0001-5403-3279
}

\section{Resumo}

Objetivo: $\mathrm{Na}$ ausência de mecanismos para proteger acionistas minoritários, controladores poderão exercer benefícios privados, utilizando mecanismos como as Transações com Partes Relacionadas (TPRs) ou desvios de direitos. O objetivo deste artigo é analisar os fatores explicativos das TPRs, em empresas controladoras/controladas e coligadas com estrutura piramidal no Brasil.

Método: Os dados de TPRs foram obtidos por meio do Formulário de Referência para 153 empresas no período de 2010 a 2017. Mediante a estimação de regressões quantílicas, buscou-se encontrar os fatores (presença de estrutura piramidal, desempenho, valor da firma e governança corporativa) que mais explicam essas transações, tanto entre controladoras/controladas quanto entre coligadas.

Resultados: Para controladoras/controladas, os fatores explicativos das TPRs são os desvios de direitos, alavancagem, presença de acionistas estrangeiros e auditores independentes. Para coligadas, constataramse efeitos da rentabilidade do ativo (ROA), tangibilidade e auditoria pelas Big Four.

Contribuições: $\mathrm{O}$ estudo contribui ao demonstrar que há um efeito da estrutura piramidal nas TPRs sem empresas controladoras/controladas e do desempenho nas coligadas, sendo que a governança não foi um moderador para mitigar conflitos de interesses. Portanto, abordaram-se temáticas pouco exploradas na literatura nacional e nos mercados emergentes, caracterizados por estruturas de propriedade concentradas, permitindo novos caminhos para as relações de agência.

Palavras-chave: Transações com partes relacionadas; Estrutura piramidal; Empresas controladoras/ controladas; Empresas coligadas. 


\section{Introdução}

Sob a ótica da teoria da Agência, na ausência de mecanismos para proteger os acionistas minoritários, os controladores poderão exercer benefícios privados (Cho \& Lim, 2018). Esses podem usar alguns métodos para maximizar seus interesses, como, por exemplo, as transações com partes relacionadas e os desvios de direitos e, consequentemente, expropriar acionistas minoritários (Cheung, Jing, Lu, Rau, \& Stouraitis, 2009). As TPRs podem ser entendidas como laços de negócios, pois correspondem a operações (ativos, bens, capital próprio, entre outros) com acionistas, membros do conselho de administração e empresas afiliadas ou subsidiárias (Cho \& Lim, 2018).

As TPRs são comumente realizadas entre empresas afiliadas a business groups. Já que os grupos costumam se organizar em estruturas de propriedade piramidal, formando family business groups (Almeida \& Wolfenzon, 2006; Bena \& Ortiz-Molina, 2013; Claessens, Djankov, \& Lang, 2000), as TPRs são analisadas de acordo com a posição das empresas na estrutura. Entre essas posições, têm-se as controladoras/ controladas e coligadas. As sociedades coligadas são aquelas possuidoras de participação acionária significativa em outras sociedades, sem, contudo, controlá-las. Já as sociedades controladoras/controladas se caracterizam pela relação de subordinação entre si, por meio da propriedade das ações (Almeida, 1987).

Paralelo a isso, outra forma de expropriação de acionistas minoritários corresponde aos desvios de direitos da estrutura piramidal. Esses são entendidos como a diferença entre direito de voto e direito de fluxo de caixa (Kang, Lee, Lee, \& Park, 2014). Nas estruturas piramidais, uma empresa (que pode ser também um indivíduo, uma família ou o Governo) obtém o controle de múltiplas empresas por meio de uma relação hierárquica de propriedade (Almeida \& Wolfenzon, 2006). Nessas estruturas de propriedade, o acionista controlador exerce o controle mediante, pelo menos, uma empresa listada publicamente (La Porta, Lopez-de-silanes, \& Shleifer, 1999).

A visão tradicional para a formação de estruturas piramidais está pautada na tentativa de maximizar o desvio entre direitos de voto e de fluxo de caixa, a partir de níveis intermediários de empresas, gerando diferenças entre o controle e a propriedade (Aldrighi \& Mazzer Neto, 2005). Portanto, os desvios de direitos e as transações com partes relacionadas podem ser canais de expropriação dos acionistas minoritários. A relação entre esses conceitos baseia-se no pressuposto de que a dominância nos direitos de voto, ocasionada pelos desvios, poderá garantir aos acionistas controladores uma maior capacidade e flexibilidade de se envolverem em TPRs para seus interesses pessoais (Rahmat, Amin, \&Saleh, 2018). Tendo em vista esses argumentos, tem-se como objetivo geral analisar os fatores explicativos das TPRs, em empresas controladoras/controladas e coligadas com estrutura piramidal no Brasil.

Esse estudo se justifica pela investigação das transações com partes relacionadas em empresas com estrutura de propriedade indireta, uma temática pouco explorada na literatura nacional. Portanto, cabe analisar os fatores explicativos dessas transações, considerando-se as posições das empresas (controladoras/controladas ou coligadas) na estrutura piramidal. Ainda, a aplicação em um mercado emergente é relevante, visto que as TPRs estão prevalecendo nessas economias, devido a falhas na governança corporativa e presença generalizada de grupos controlados por famílias via estrutura piramidal e participações cruzadas (Wang, Cho, \& Lin, 2019).

Para analisar a relação entre TPRs e estrutura piramidal, foram obtidos dados do Formulário de Referência para 153 empresas listadas na bolsa de valores. Foram estimadas regressões quantílicas no período de 2010 a 2017. De forma geral, os resultados reportaram que a estrutura piramidal é um fator explicativo para as TPRs nas operações que envolvem empresas controladoras/controladas. Porém, para as empresas coligadas, não houve influência estatisticamente significativa dos desvios de direitos nas TPRs. Quanto aos demais resultados, verificou-se que o desempenho da firma e a governança corporativa tiveram influência nas TPRs com coligadas. Porém, no modelo de controladoras/controladas, a governança corporativa não reduz o valor transacionado entre essas empresas. 
A pesquisa avança teoricamente no contexto das relações de agência, a partir das diferenças entre estrutura de controle e estrutura de propriedade (desvios). Propôs-se examinar as TPRs nas estruturas piramidais, considerando o efeito dos vínculos societários. Grande parte dos estudos analisa as transações de forma ampla. Portanto, a contribuição teórica consiste em demonstrar que a subordinação existente entre empresas controladoras/controladas poderá estimular a utilização de TPRs. Os resultados são relevantes por evidenciarem uma associação positiva entre TPRs e níveis de desvio de direitos, ratificando a hipótese dos conflitos de interesses, nos quais acionistas controladores poderão utilizar transações para obter benefícios privados. Em função disso, as TPRs e os desvios de direitos podem ser considerados canais de expropriação de acionistas minoritários. Nesse sentido, quanto maior o nível de desvios (mensurado pela regressão quantílica), maior a probabilidade de a empresa utilizar TPRs para negociar com controladoras/controladas.

Este artigo está estruturado em cinco seções, iniciando por esta introdução. Na segunda parte, expõe-se a fundamentação teórica, com questões relativas às transações com partes relacionadas e estrutura piramidal. A terceira seção expõe a metodologia de pesquisa e, na quarta seção, são analisados os resultados. Por fim, na última seção, são apresentadas as considerações finais com apontamentos de algumas reflexões que contribuam para o melhor entendimento das temáticas abordadas.

\section{Transações com partes relacionadas em estruturas piramidais: concepções e hipóteses}

\subsection{Transações com Partes Relacionadas}

As TPRs são comumente realizadas entre empresas afiliadas a business groups, conceituadas pela sociologia econômica como um conjunto de empresas separadas legalmente, mas que estão vinculadas por relações persistentes e/ou informais (Granovetter, 2005). Para o contexto brasileiro, business groups se referem a um grupo de empresas que, além de serem estritamente controladas pela mesma entidade, abrangem, pelo menos, uma empresa listada no mercado de capitais ('listed-firm business groups') ou apresentam diversificação de unidades de negócios ('diversified business groups') em pelo menos três setores (Aldrighi \& Postali, 2010).

Com isso, a literatura reconhece três motivações para as TPRs: tunneling, propping e gerenciamento de resultados (Cheunget al., 2009). O conceito de tunneling foi introduzido por Johnson, La Porta, Lopezde-Silanes, e Shleifer (2000), sendo uma importante contribuição para a temática das TPRs. Conforme os autores, o tunneling compreende a transferência de recursos de uma empresa na base da pirâmide para as que estão nos níveis superiores, aumentando os ganhos pessoais dos controladores (Johnson, La Porta, Lopezde-Silanes, \& Shleifer, 2000). O inverso também ocorre, sendo chamado de propping e tendo por objetivo auxiliar as empresas que estão na base da pirâmide (Bertrand \& Mullainathan, 2003; Jian \& Wong, 2010). Já as escolhas contábeis agressivas e a manipulação de lucros estão relacionadas ao gerenciamento de resultados, que, nas TPRs, pode ser oriundo das vendas em dinheiro entre partes relacionadas (Jian \& Wong, 2010).

No Brasil, a regulamentação das TPRs está pautada no pronunciamento técnico CPC n. ${ }^{\circ}$ 5/R1 (CVM, 2010, p.4), que conceitua as transações como "transferências de recursos, serviços ou obrigações entre uma entidade que reporta a informação e uma parte relacionada, independentemente de ser cobrado um preço em contrapartida". No CPC n. ${ }^{\circ}$ /R1, são mencionados alguns exemplos de transações que devem ser divulgadas, tais como: (i) compras ou vendas de bens (acabados ou não acabados); (ii) compras ou vendas de propriedades e outros ativos; (iii) prestação ou recebimento de serviços; (iv) arrendamentos; (v) transferências de pesquisa e desenvolvimento; (vi) transferências mediante acordos de licença; (vii) transferências de natureza financeira (incluindo empréstimos e contribuições para capital em dinheiro ou equivalente); (viii) fornecimento de garantias, avais ou fianças; (ix) assunção de compromissos para o caso de um evento particular ocorrer ou não no futuro, incluindo contratos a executar (reconhecidos ou não); e (x) liquidação de passivos em nome da entidade ou pela entidade em nome de parte relacionada. 
Para que os usuários de demonstrações contábeis tenham uma visão acerca dos efeitos dos relacionamentos entre as partes, a companhia deve divulgar as transações que tenham valor total maior que R $\$ 6$ milhões, 1\% do ativo total da companhia ou que, no entender dos administradores, sejam consideradas relevantes. Para isso, devem detalhar informações, como o montante da transação e os saldos existentes, incluindo prazos e condições, e a relação entre a entidade que reporta a informação com a respectiva parte relacionada, além de outros fatores que caracterizem a transação, segundo a Comissão de Valores Mobiliários [CVM] (CVM, 2010).

No caso específico das TPRs e o valor da empresa, duas vertentes concorrentes são abordadas na literatura (Bona-Sánchez, Fernández-Senra, \& Pérez-Alemán, 2017; Kang et al., 2014). A primeira delas é denominada hipótese de transações eficientes, a qual evidencia que as TPRs podem minimizar os custos de transação e contribuir positivamente para o valor da firma (Gordon, Henry, \& Palia, 2004). Por outro lado, na hipótese de conflitos de interesses, essas TPRs podem reduzir o valor da firma, em função dos conflitos entre acionistas majoritários e minoritários (Gordon et al., 2004; Johnson et al., 2000). A seguir, é evidenciado como essas TPRs podem ser influenciadas pelas estruturas piramidais.

\subsection{Estruturas Piramidais}

As estruturas piramidais existem pelo controle indireto de uma corporação exercido por outra corporação (Tirole, 2006), sendo as discrepâncias entre direitos de controle e propriedade a principal forma de mensurá-las (Aldrighi \& Postali, 2011). São estruturas mais comuns em países com fraca proteção aos acionistas, possibilitando a criação de novas empresas por meio de controle piramidal (Almeida \& Wolfenzon, 2006). A cadeia de propriedades formada pelas pirâmides permite que o proprietário final tenha o controle de todas as empresas, inclusive aquelas em que ele não possui nenhuma propriedade direta (Bertrand \& Mullainathan, 2003).

A visão tradicional para a formação de estruturas piramidais está pautada na tentativa de maximizar o desvio entre direitos de voto e de fluxo de caixa, a partir de níveis intermediários de empresas, gerando diferenças entre controle e propriedade (Aldrighi \& Mazzer Neto, 2005). Os direitos de voto são oriundos das ações ordinárias, determinando o controle da empresa, enquanto que os direitos sobre o fluxo de caixa são resultado da quantidade de ações do acionista, com ou sem direito de voto (Bortolon, 2010). Assim, o excesso de direitos de voto pode aumentar o poder dos grandes acionistas e, consequentemente, representar o potencial de expropriação dos acionistas minoritários (Aldrighi \& Mazzer Neto, 2005; La Porta et al., 1999).

Almeida e Wolfenzon (2006) desenvolveram um modelo teórico alternativo, para explicar a formação de estruturas piramidais, sendo um dos trabalhos mais referenciados na literatura sobre o tema. Conforme os autores, a criação de pirâmides tem como características as empresas com necessidade de altos níveis de investimento e/ou baixa rentabilidade. A vantagem do financiamento permite que as famílias, que já controlam outras empresas publicamente, desenvolvam novos empreendimentos, possuindo, indiretamente, ações dessa nova empresa. Essa estrutura piramidal pode permitir que as famílias obtenham benefícios desse novo 'financiamento', o qual não parecerá rentável para investidores externos (Almeida \& Wolfenzon, 2006).

As estruturas piramidais são muito comuns em alguns países, como a Europa Continental, Ásia e América do Sul, frequentemente, organizando-se em business groups familiares (Claessens et al., 2000). Portanto, a interconexão entre business groups e estruturas piramidais está nas relações de propriedade das firmas, já que as pirâmides são a principal forma de organização desses grupos (Almeida \& Wolfenzon, 2006). 
O Brasil é um caso interessante a ser estudado em função da estrutura do mercado de ações (emissão de duas classes) e alterações nas normas de governança corporativa. De acordo com a legislação, as empresas podem emitir ações ordinárias nominativas $(\mathrm{ON})$ e ações preferenciais nominativas $(\mathrm{PN})$. A primeira classe de ações possui direito a voto, enquanto a segunda pressupõe preferência no recebimento de dividendos e no reembolso de capital, em caso de dissolvência da companhia. Com isso, as empresas poderão utilizar as duas classes de ações nas estruturas piramidais para manter seu poder de voto e buscar investimentos no mercado de capitais. A emissão de duas classes de ações e as estruturas piramidais são consideradas técnicas para o desvio do princípio "uma ação, um voto" (La Porta et al., 1999; La Porta, Lopez-de-silanes, Shleifer, \& Vishny, 2000).

Nesse caso, as estruturas piramidais poderão utilizar TPRs para obter benefícios ao longo da cadeia hierárquica, a qual é formada por vínculos societários. Entre esses vínculos, tem-se a presença de empresas controladoras/controladas e coligadas. Souza, Knupp e Borba (2013) evidenciaram que o maior número de empresas coligadas e controladas resulta em maior valor envolvido em transações com partes relacionadas. Para os autores, esse fato sugere que as TPRs podem ter propósitos estratégicos ou financeiros, os quais estão vinculados à natureza societária existente, como a participação acionária (coligadas) ou a influência de controle (controladoras/controladas). Portanto, espera-se que o maior desvio de direitos, oriundo da estrutura piramidal, irá influenciar na execução de TPRs. Partindo-se desse contexto, foi elaborada a seguinte hipótese de pesquisa:

Hipótese 1: Há uma associação positiva entre TPRs com controladoras/controladas e/ou coligadas e o desvio de direitos das estruturas piramidais.

Nesse contexto, a relação entre TPRs e estruturas piramidais está na opção de os acionistas controladores utilizarem as transações para obter benefícios privados ao longo da cadeia de propriedade. Com isso, a estrutura piramidal pode ser um incentivo às TPRs, havendo uma associação positiva entre ambos (Kang et al., 2014; Maheshwari \& Gupta, 2018).

O incentivo às TPRs também pode estar relacionado com o desempenho e valor da firma. Resultados empíricos têm demonstrado que as transações com partes relacionadas estão negativamente associadas ao valor da firma, em função dos conflitos de interesses (Bona-Sánchez et al., 2017; Cheung, Jing, et al., 2009). Também, negociações com partes relacionadas mais elevadas estão associadas ao pior desempenho da empresa (Wang et al., 2019), já que essas companhias poderão ter incentivos para ganhos oportunistas (Kang et al., 2014).

Por outro lado, TPRs entre empresas do mesmo grupo podem influenciar positivamente o valor da firma (Wong, Kim, \& Lo, 2015), assim como empresas com maior similaridade e integração vertical no grupo podem obter melhor desempenho a partir de TPRs (Wang et al., 2019). A organização de empresas em grupos pode reduzir custos de transação e permitir a formação de mercados internos. Os mercados internos facilitam os inter-relacionamentos entre as empresas afiliadas, proporcionando economias de escopo e melhor alocação de recursos, o que pode gerar melhor desempenho ao realizar TPRs (Wang et al., 2019). Maheshwari e Gupta (2018), por exemplo, encontraram uma associação positiva entre TPRs e desempenho, confirmando a sinergia que pode existir nos mercados internos. Porém, de forma geral, a literatura aponta que TPRs são geralmente associadas com baixo desempenho e valor da firma. Em função disso, foram elaboradas as seguintes hipóteses de pesquisa:

Hipótese 2: Há uma associação negativa entre TPRs com controladoras/controladas e/ou coligadas e o valor da firma.

Hipótese 3: Há uma associação negativa entre TPRs com controladoras/controladas e/ou coligadas e o desempenho. 
Outro aspecto reportado na literatura, como possível fator explicativo das TPRs, é a governança corporativa. Considerando que a estrutura de controle e a propriedade podem determinar a governança corporativa das empresas, espera-se que o aumento do nível de proteção ao investidor resulte em menor probabilidade de haver estruturas piramidais (Almeida \& Wolfenzon, 2006) e TPRs (Kang et al., 2014). No caso das TPRs, a maior parte da literatura demonstrou que TPRs são mais prováveis quando os mecanismos de governança corporativa são fracos (Bhuiyan \& Roudaki, 2018; Kang et al., 2014; Rahmat et al., 2018). Em função disso, inseriu-se a seguinte hipótese de pesquisa:

Hipótese 4: Há uma associação negativa entre TPRs com controladoras/controladas e/ou coligadas e governança corporativa.

Assim, de forma geral, têm-se os principais aspectos relacionados às TPRs que foram analisados na pesquisa. Com base nas TPRs entre empresas do mesmo grupo, torna-se relevante analisar o efeito dessas operações nas controladoras/controladas e coligadas. Com isso, podem-se identificar os fatores explicativos para TPRs conforme os vínculos societários existentes. Na próxima seção, tem-se o método.

\section{Método}

\subsection{Caracterização da amostra e variáveis utilizadas}

Para identificar as estruturas piramidais, foram acessados os dados anuais das empresas brasileiras listadas na $[\mathrm{B}]^{3}$, via formulário de referência, no período de 2010 a 2017. A extração dos dados foi realizada através do pacote GetDFPData (Perlin, Kirch, \& Vancin, 2019) do software R, o qual possibilita acesso às informações das demonstrações financeiras das empresas e ao formulário de referência da CVM. Quanto a esse último, é possível incluir filtros, elencando os itens do formulário que se busca acessar.

Para selecionar a amostra, utilizou-se, como critério, a definição de estruturas piramidais, exposta por La Porta et al. (1999), os quais caracterizam as pirâmides como aquelas empresas em que o acionista controlador exerce o controle por meio de, pelo menos, uma empresa de capital aberto. O processo de amostragem resultou em um painel não balanceado, com 961 observações e 155 empresas, sendo ajustado conforme o critério de exclusão de empresas/ano com Q de Tobin negativo e superior a 10 (Kirch, Procianoy, \& Terra, 2014). Assim, a amostra final é composta por 929 observações e 153 empresas com estrutura piramidal no Brasil. Dessas, foram selecionadas as transações com partes relacionadas realizadas com suas controladoras/controladas e coligadas.

Foram utilizadas, como variáveis dependentes, o valor total das TPRs com controladoras/controladas e com coligadas, pois sua magnitude pode representar maior potencial de tunneling (Chen, Li, \& Chen, 2017). Já para a adequação ao modelo, realizou-se o ajuste inflacionário e aplicação do logaritmo natural aos valores totais estudados (Silveira, Prado, \& Sasso, 2008). 
Para representar a estrutura de controle e propriedade das pirâmides, calculou-se o desvio de direitos. Ele é encontrado a partir das cadeias de propriedades existentes e resulta da diferença entre a parcela de direitos de voto e a parcela de direitos de fluxo de caixa. Portanto, iniciou-se a coleta com a identificação dos acionistas diretos de cada empresa, filtrando-se apenas os de natureza jurídica. Ao verificar a existência de um acionista jurídico listado, a empresa foi incluída na amostra e foram identificados os seus acionistas diretos, que são, portanto, acionistas indiretos da empresa da amostra. Esse procedimento foi repetido até alcançar os acionistas últimos de cada cadeia de propriedade.A parcela dos direitos de fluxo de caixa foi calculada pelo produto das participações acionárias (total de ações) nas empresas ao longo da cadeia (Aldrighi, 2014; Bortolon, 2010). Já, a parcela de direitos de voto na propriedade indireta depende da existência do controle. Se o maior acionista último (MAU) for controlador (possuir pelo menos 50\% dos direitos de voto), a parcela de direitos de voto é igual à participação direta que o último intermediário na cadeia de propriedade detém no capital votante da empresa analisada. Se o MAU não for controlador, o cálculo é idêntico à parcela de direitos de fluxo de caixa, ou seja, o produto das participações (Aldrighi, 2014).

Para identificar o efeito do desempenho e valor das firmas nas TPRs, foram incluídos o retorno sobre o ativo (ROA) e o Q de Tobin, os quais são medidas amplamente usadas na literatura para esse fim (Kang et al., 2014). Quanto ao sinal esperado dessas variáveis em relação às TPRs, pode ser positivo ou negativo. Empresas com elevado desempenho e valor poderão utilizar as TPRs como forma de manter/ aumentar seus resultados, sob a ótica das transações eficientes, as quais reduzem os custos (Wong et al., 2015). Por outro lado, empresas que possuem restrições financeiras serão mais suscetíveis a utilizar esse tipo de operação para obter ganhos oportunistas (Kang et al., 2014; Wang et al., 2019).

Já a governança corporativa foi analisada por ser uma forma de mitigar a expropriação dos acionistas minoritários. Nesse caso, foram incluídas três formas distintas de mensurar a governança, a partir de variáveis dummies: (1) adesão da empresa ao segmento diferenciado do Novo Mercado; (2) presença de membros independentes no comitê de auditoria (Kang et al., 2014; Rahmat et al., 2018); e, (3) realização de auditoria externa por uma das quatro maiores empresas do ramo (Deloitte, Ernst \& Young, KPMG ou PricewaterhouseCoopers - PwC) (Bhuiyan \& Roudaki, 2018; Rahmat et al., 2018).

Para complementar a análise, foram incorporadas variáveis de controle. O "tamanho da empresa" foi selecionado, devido à possibilidade de empresas maiores terem um volume mais representativo de TPRs (Kang et al., 2014). A alavancagem está relacionada com as questões de desempenho e monitoramento por parte dos credores. Empresas com posições financeiras vulneráveis tenderão a participar de TPRs para superar suas dificuldades (Bhuiyan \& Roudaki, 2018). Por outro lado, a alavancagem pode implicar maior monitoramento por partes dos credores (Aldrighi, 2014), situação que poderá reduzir a execução de TPRs (Matos \& Galdi, 2014).

Quanto à tangibilidade, nas TPRs, os ativos imobilizados podem servir como garantias e, por isso, espera-se uma relação positiva entre a tangibilidade e maior ocorrência desses contratos. Ativos intangíveis são mais difíceis de monitorar e, consequentemente, podem estar sujeitos às decisões gerenciais (Himmelberg, Hubbard, \& Palia, 1999), facilitando as TPRs. Referente ao capital estrangeiro, espera-se que empresas que possuem acionistas estrangeiros pratiquem mais transações entre si (Cheung et al., 2009). 
A Tabela 1 mostra as definições das variáveis utilizadas na pesquisa, iniciando pelas transações com partes relacionadas, as quais representam as variáveis dependentes. Após, são descritos os fatores explicativos para TPRs, que compreendem a estrutura piramidal (desvios de direitos), o desempenho, o valor da firma e a governança corporativa. Essas relações foram mediadas por variáveis de controle relacionadas às temáticas, incluindo-se medidas importantes para a análise dos resultados, tais como tamanho e alavancagem. As variáveis foram selecionadas e descritas com base na literatura apresentada nas referências. Na sequência, descreve-se o modelo de regressão utilizado.

Tabela 1

\section{Variáveis da Pesquisa}

\begin{tabular}{|c|c|c|c|}
\hline Variável e Sigla & Medida & Referências & \\
\hline $\begin{array}{l}\text { Valor total das TPRs } \\
\text { com controladoras/ } \\
\text { controladas (LVC) }\end{array}$ & Log (LVC) por empresa/ano & \multirow{2}{*}{$\begin{array}{l}\text { Silveira et al. (2008); Matos } \\
\text { e Galdi (2014) }\end{array}$} & \\
\hline $\begin{array}{l}\text { Valor total das TPRs } \\
\text { com coligadas (LVCOL) }\end{array}$ & Log (LVCOL) por empresa/ano & & \\
\hline Variável e Sigla & Medida & Referências & SE \\
\hline Desvio (LD) & $\begin{array}{l}\text { Log }(D) \text {, sendo D= DVMC-DFC } \\
\text { Em que: DVMC: direito de voto pela existência (ou } \\
\text { não) do MAU controlador }\end{array}$ & $\begin{array}{l}\text { Aldrighi (2014); Aldrighi et al. } \\
\text { (2018) }\end{array}$ & $(+)$ \\
\hline $\begin{array}{l}\text { Rentabilidade do Ativo } \\
\text { (ROA) }\end{array}$ & $\mathrm{ROA}=\frac{\text { Resultado Operacional }}{\text { AtivoTotal }}$ & $\begin{array}{l}\text { Kang et al. (2014); Maheshwari } \\
\text { e Gupta (2018); Wang et al. } \\
\text { (2019) }\end{array}$ & \multirow[b]{2}{*}{$\begin{array}{l}(+) \\
\text { ol } \\
(-)\end{array}$} \\
\hline Q de Tobin (QT) & $\begin{array}{l}\text { QT = } \frac{V M A O+V M A P+D I V T}{A t i v o T o t a l} \\
\text { Em que: VMAO: valor de mercado das ações } \\
\text { ordinárias; VMAP: valor de mercado das ações } \\
\text { preferenciais; DIVT: valor contábil do passivo } \\
\text { exigível (circulante + não circulante) menos o ativo } \\
\text { circulante, após a exclusão dos estoques }\end{array}$ & $\begin{array}{l}\text { Bona-Sánchez et al. (2017); } \\
\text { Maheshwari e Gupta (2018) }\end{array}$ & \\
\hline $\begin{array}{l}\text { Presença no Novo } \\
\text { Mercado (NM) }\end{array}$ & $\begin{array}{l}\text { Variável binária igual a (1), se a empresa participa do } \\
\text { Novo Mercado; e (0), caso contrário }\end{array}$ & Aldrighi et al. (2018) & \multirow{3}{*}{$(-)$} \\
\hline Big Four (Big4) & $\begin{array}{l}\text { Variável binária igual a (1), se a empresa é auditada } \\
\text { pelas } 4 \text { grandes empresas de auditoria; e (0), caso } \\
\text { contrário }\end{array}$ & $\begin{array}{l}\text { Bhuiyan e Roudaki (2018); } \\
\text { Rahmat et al. (2018) }\end{array}$ & \\
\hline $\begin{array}{l}\text { Auditoria independente } \\
\text { (Audit) }\end{array}$ & $\begin{array}{l}\text { Variável binária igual a (1), se a empresa possui } \\
\text { membros independentes no comitê de auditoria; e } \\
\text { (0), caso contrário }\end{array}$ & $\begin{array}{l}\text { Kang et al. (2014); Rahmat et al. } \\
\text { (2018) }\end{array}$ & \\
\hline Tamanho (LRCL) & $\log (R C L)$, sendo $R C L=$ receita líquida de vendas & Kang et al. (2014). & $(+)$ \\
\hline $\begin{array}{l}\text { Alavancagem em } \\
\text { relação ao Ativo Total } \\
\text { (Alav) }\end{array}$ & ALAV $=\frac{\text { Passivo Circulante }+ \text { Passivo Não Circulante }}{\text { AtivoTotal }}$ & $\begin{array}{l}\text { Aldrighi (2014); Aldrighi et al. } \\
\text { (2018); Maheshwari e Gupta } \\
\text { (2018) }\end{array}$ & $\begin{array}{l}(+) \\
\text { ou } \\
(-)\end{array}$ \\
\hline Tangibilidade (Tangib) & TANGIB $=\frac{\text { Ativolmobilizado }}{\text { AtivoTotal }}$ & $\begin{array}{l}\text { Souza e Bortolon (2014); } \\
\text { Aldrighi et al. (2018) }\end{array}$ & $\begin{array}{l}(+) \\
\text { ou } \\
(-)\end{array}$ \\
\hline $\begin{array}{l}\text { Capital Estrangeiro } \\
\text { (Estrang) }\end{array}$ & $\begin{array}{l}\text { Variável binária igual a (1), se há ações ordinárias e/ } \\
\text { ou preferenciais sob posse de capital estrangeiro; e } \\
\text { (0), caso contrário }\end{array}$ & Cheung et al. (2009) & $(+)$ \\
\hline Efeitos Fixos Temporais & $\begin{array}{l}\text { Variável binária em que } 1 \text { indica o ano em que o } \\
\text { dado é gerado e } 0 \text { caso contrário }\end{array}$ & $\begin{array}{l}\text { Kang et al. (2014); Bona-Sánchez } \\
\text { al. (2017) }\end{array}$ & \\
\hline
\end{tabular}

Legenda: *a formação das variáveis quantitativas para as TPRs considerou o período de referência divulgado pelas empresas e não especificamente a data da transação, visto que muitas transações se mantêm ativas por longo prazo. SE (sinal esperado) corresponde ao tipo de associação da variável dependente com a variável independente. O sinal esperado é definido com base na literatura empírica prévia.

Fonte: elaborado pelos autores (2021). 


\subsection{Modelo de Regressão Quantílica}

O modelo utilizado é derivado do estimador de Machado e Santos Silva (2019), desenvolvido para dados em painel com efeitos fixos, considerando especificações lineares e não lineares. Optou-se pela estimação linear, na qual os efeitos individuais podem afetar toda a distribuição, a partir dos quantis condicionais. Assim, os dados em painel com efeitos individuais, considerando a estimação com quantis condicionais para as distribuições de probabilidade (localização e escala), têm o padrão descrito na Equação (1).

$$
Y_{i t}=\alpha_{i}+X_{i t}^{\prime} \beta+\left(\delta_{i}+Z_{i t}^{\prime} \gamma\right) U_{i t}
$$

Com $\operatorname{Pr}\left\{\delta_{\mathrm{i}}+Z_{i t}^{\prime} \gamma>0\right\}=1$. Os parâmetros $\left(\alpha_{\mathrm{i}}, \delta_{\mathrm{i}}\right)$, capturam o efeito fixo individual $(i)$ e $Z$ é definido antes. A sequência $\left\{X_{i t}\right\}$ é estritamente exógena, i.i.d para qualquer $i$ fixo, e independente entre $i . U_{i t}$ é i.i.d (através de $i$ e $t$ ), estatisticamente independente de $X_{i t}$ e normalizado para satisfazer as condições de momento. O modelo 1 implica a Equação 2.

$$
Q_{\gamma}\left(\tau \mid X_{i t}\right)=\left(\alpha_{i}+\delta_{i} q(\tau)\right)+X_{i t}^{\prime} \beta+Z_{i t}^{\prime} \gamma q(\tau)
$$

Em que $\alpha_{i}(\tau) \equiv \alpha_{i}+\delta_{\mathrm{i}} q(\tau)$ é chamado de coeficiente escalar do efeito fixo por quantil $(\tau)$ para o indivíduo $i$ ou o efeito de distribuição em $(\tau)$. O efeito de distribuição difere do efeito fixo usual, pois não é, em geral, um deslocamento. Ou seja, o efeito de distribuição representa o efeito de características individuais invariantes no tempo que, como outras variáveis, pode ter impactos diferentes em distintas regiões de distribuição condicional $Y$. O fato de $\int_{0}^{1} q(\tau) \mathrm{d} \tau=0$ implica que $\alpha_{i}$ possa ser interpretado como o efeito médio para o indivíduo $i$. Dessa forma, a regressão quantílica para painel de dados com efeitos fixos é, então, estimada via momentos (MM-QR), permitindo relações dinâmicas que trabalham com condições de ortogonalidade (Hansen, 1982).

Nesse sentido, por ser um modelo robusto, a regressão quantílica para dados em painel com efeitos fixos foi estimada para entender os fatores explicativos das TPRs nas empresas controladoras/controladas e coligadas. Portanto, assim como no painel tradicional, as TPRs representam a variável dependente, que corresponde ao valor total das transações com partes relacionadas ao longo do tempo. As principais variáveis independentes se referem aos desvios de direitos, desempenho, valor da firma e governança corporativa. E, por fim, foram incluídas as variáveis de controle, que contribuem para a interpretação dos coeficientes.

A utilização da regressão quantílica justifica-se pela necessidade de analisar o efeito de cada nível de desvio de direitos nos respectivos níveis de valores das TPRs. Espera-se que maiores níveis de desvios possam estimular um maior volume ou valor de transações. Além disso, na regressão quantílica, podem ser usados outliers, porque o método é robusto para esses valores. No que se refere ao estimador escolhido para a regressão quantílica (Machado \& Santos Silva, 2019), não se encontrou aplicação no Brasil, sendo apropriado ao painel de dados e recentemente desenvolvido, o que caracteriza uma robustez e originalidade ao estudo.

Relativo ao ajuste, os modelos lineares tradicionais utilizam como parâmetro o coeficiente de determinação $\left(\mathrm{R}^{2}\right)$. Essa estatística pode ser entendida como o percentual de variabilidade da variável resposta explicada pelas variáveis independentes. Na regressão quantílica, são estimados os pseudos $\mathrm{R}^{2}$ para cada um dos quantis. Porém, essa medida pode não ser adequada, indicando-se o teste de erro de especificação da regressão para analisar o ajuste da regressão quantílica com dados em painel e efeitos fixos (Machado \& Santos Silva, 2019).

O teste RESET (Regression Specification Error Test) proposto por Ramsey (1969) tem como objetivo identificar erros de especificação na regressão. No RESET, é comparado um modelo nulo com um alternativo, que é uma generalização não declarada desse modelo. Assim, busca descobrir não linearidades em sua forma funcional (Greene, 2012). A hipótese nula estabelece que o modelo está corretamente estimado. Portanto, aceitando-se a hipótese nula ( $p$-value $>0,05)$, tem-se que o modelo é válido, sendo possível interpretar seus coeficientes. A seguir, são apresentados os resultados e as discussões do trabalho. 


\section{Resultados e Discussões}

Para caracterizar as empresas da amostra, foram analisados os setores de atuação, conforme classificação da CVM (bens industriais; consumo cíclico; consumo não cíclico; financeiro e outros; materiais básicos; petróleo, gás e biocombustíveis; saúde; tecnologia da informação; telecomunicações; e utilidade pública). Identificou-se que os três setores mais representativos correspondem à utilidade pública $(32,40 \%)$, financeiro e outros $(20,02 \%)$ e bens industriais $(18,57 \%)$. Previamente à aplicação da regressão quantílica, a estatística descritiva foi analisada, conforme exposto na Tabela 2.

Tabela 2

\section{Estatística Descritiva das Variáveis}

\begin{tabular}{crrrrrrrrr}
\hline $\begin{array}{c}\text { Variável/ } \\
\text { Estatística }\end{array}$ & Média & Mediana & $\begin{array}{c}\text { Desvio } \\
\text { Padrão }\end{array}$ & Mínimo & Máximo & p10 & p25 & p75 & p90 \\
\hline VT CT* & 4,29 & 0,26 & 9,36 & 0,00 & 36,10 & 0,00 & 0,03 & 1,68 & 16,40 \\
\hline LN CT & 19,40 & 19,47 & 2,98 & 12,89 & 24,32 & 15,33 & 17,68 & 21,33 & 23,61 \\
\hline VT CG* & 2,32 & 0,03 & 6,61 & $-0,03$ & 26,00 & 0,00 & 0,00 & 0,36 & 5,62 \\
\hline LN CG & 17,75 & 17,77 & 3,56 & 10,32 & 24,20 & 12,46 & 15,59 & 19,81 & 22,82 \\
\hline Desvio & 0,31 & 0,19 & 0,34 & 0,00 & 0,94 & 0,00 & 0,00 & 0,61 & 0,90 \\
\hline ROA & 0,05 & 0,05 & 0,11 & $-0,22$ & 0,27 & $-0,08$ & 0,01 & 0,12 & 0,22 \\
\hline Qtobin & 0,95 & 0,77 & 0,69 & 0,07 & 3,05 & 0,27 & 0,56 & 1,10 & 1,87 \\
\hline RCL* & 2,80 & 0,59 & 4,49 & 0,00 & 16,80 & 0,00 & 0,02 & 3,63 & 8,70 \\
\hline ALAV & 0,53 & 0,58 & 0,26 & 0,02 & 0,95 & 0,13 & 0,33 & 0,74 & 0,87 \\
\hline Tang & 0,11 & 0,00 & 0,19 & 0,00 & 0,65 & 0,00 & 0,00 & 0,16 & 0,46 \\
\hline
\end{tabular}

Legenda: o * representa valores em bilhões de reais. VT CT: valor total em reais das transações com partes relacionadas correspondentes a empresas controladoras e/ou controladas; LN CT: logaritmo natural do valor total em reais das transações com partes relacionadas correspondentes a empresas controladoras e/ou controladas; VT CG: valor total em reais das transações com partes relacionadas correspondentes a empresas coligadas; LN CG: logaritmo natural do valor total em reais das transações com partes relacionadas correspondentes a empresas coligadas. ROA: rentabilidade do ativo total; RCL: receita líquida de vendas/serviços; ALAV: alavancagem pelo ativo total; Tang: tangibilidade do ativo.

Fonte: dados da pesquisa.

De maneira geral, a Tabela 2 evidencia que a média do valor total das transações para controladoras/ controladas é de $\mathrm{R} \$ 4,29$ bilhões e para coligadas é R \$2,32 bilhões. Para complementar a descrição, utilizaramse essas variáveis em logaritmo natural, a fim de incluí-las no modelo de dados em painel. Nesses dados, a média e a mediana são muito próximas, demonstrando adequação das informações para os testes realizados.

As informações sobre o desempenho mostram que os dados também apresentaram consistência, pela proximidade da média e mediana. Em termos interpretativos, em média, as empresas apresentam uma rentabilidade positiva de $6 \%$. Sobre o valor da firma, mensurado pelo Q de Tobin, observa-se que o valor de mercado representa $95 \%$ do valor patrimonial. Em termos de alavancagem, o capital de terceiros representa $53 \%$ do ativo total, sendo que o imobilizado corresponde a $11,8 \%$ dessa mesma variável. 
Após conhecer o perfil das variáveis, testou-se a correlação entre elas, constatando-se que estavam adequadas para utilização nos modelos de regressão quantílica. Os resultados são apresentados na Tabela 3.

Tabela 3

Correlação das Variáveis

\begin{tabular}{|c|c|c|c|c|c|c|c|c|}
\hline & Desvio & LN CT & LN CG & ROA & Qtobin & $\mathrm{RCL}$ & ALAV & Tang \\
\hline Desvio & 1 & & & & & & & \\
\hline LN CT & $-0,07$ & 1 & & & & & & \\
\hline LN CG & $-0,26$ & 0,45 & 1 & & & & & \\
\hline ROA & 0,17 & 0,08 & 0,00 & 1 & & & & \\
\hline Qtobin & $-0,10$ & 0,11 & 0,11 & 0,08 & 1 & & & \\
\hline $\mathrm{RCL}$ & 0,06 & 0,15 & 0,22 & 0,13 & 0,00 & 1 & & \\
\hline ALAV & 0,19 & 0,08 & 0,19 & 0,07 & 0,05 & 0,37 & 1 & \\
\hline Tang & $-0,02$ & 0,18 & 0,29 & 0,04 & $-0,05$ & 0,15 & 0,10 & 1 \\
\hline
\end{tabular}

Legenda: LN CT: logaritmo natural do valor total em reais das transações com partes relacionadas correspondentes a empresas controladoras e/ou controladas; LN CG: logaritmo natural do valor total em reais das transações com partes relacionadas correspondentes a empresas coligadas. ROA: rentabilidade do ativo total; RCL: receita líquida de vendas/ serviços; ALAV: alavancagem pelo ativo total; Tang: tangibilidade do ativo.

Fonte: dados da pesquisa.

O teste de correlação foi aplicado para verificar se as variáveis são altamente relacionadas. Os resultados mostram que a maioria das variáveis tem uma correlação baixa. Também foi testado o Fator de Inflação da Variância (VIF), em que a média não ultrapassou 5. Sendo assim, as variáveis não apresentaram multicolinearidade, possibilitando a sua utilização nos modelos de regressão quantílica.

Nesse sentido, após caracterizarem a amostra e as variáveis da pesquisa, os modelos de regressão quantílica foram estimados para as empresas controladoras/controladas e coligadas. Ficou evidenciado que, na relação entre o valor transacionado com controladoras e controladas, seus fatores explicativos são: desvios de direitos, alavancagem, auditoria e presença de acionistas estrangeiros (Tabela 4). Os coeficientes do modelo são válidos, visto o teste de adequação realizado ( $p$-value 0,3280 ). Além do mais, os desvios são significativos nos quantis de $50 \%$ e $75 \%$, sendo que um aumento de $1 \%$ nessa variável aumenta cerca de $0,01 \%$ as TPRs. A significância estatística se concentra nos quantis mais elevados, demonstrando que essas operações se tornam mais presentes à medida que aumentam os desvios entre direitos de voto e direitos de fluxo de caixa.

Esses resultados confirmam a hipótese 1 do trabalho e corroboram com a literatura (Kang et al., 2014; Maheshwari \& Gupta, 2018), demonstrando que as TPRs poderão ser um canal de expropriação a partir dos desvios de direitos. De forma prática, a organização das empresas em estruturas piramidais pode facilitar a utilização de TPRs ao longo da cadeia de subordinação entre controladoras/controladas. Sob a hipótese dos conflitos de interesses (Gordon et al., 2004), a utilização dessas transações pode ser uma forma de obter benefícios privados para as empresas e, por isso, a importância de analisar seus fatores explicativos. 
Sobre os demais aspectos, nota-se que a alavancagem possui uma associação negativa, a qual é significativa nos quantis $50 \%$ e $75 \%$. Com isso, nota-se que o aumento de $1 \%$ na alavancagem ocasiona um aumento de $0,16 \%$ nas TPRs. A influência negativa da dívida nas TPRs com controladoras/controladas também foi encontrada por Matos e Galdi (2014), justificada pelo maior monitoramento por parte dos credores. Também, pode-se atribuir tal fato à questão do desenvolvimento do mercado interno de capitais entre empresas que pertencem a grupos. As relações entre elas podem reduzir os custos de transação e aumentar a capacidade da dívida (Cai, Zeng, Lee, \& Ozkan, 2016). Porém, a alavancagem pode influenciar as TPRs até determinados níveis de valores ou ocorrer apenas em períodos de crise, quando há maior transação de recursos entre empresas (Almeida, Kim, \& Kim, 2015).

No que tange ao comitê de auditoria, os coeficientes do modelo são os de maior impacto, significativos em quase todos os quantis (exceto no de 10\%). Nesse sentido, o valor transacionado entre essas partes relacionadas aumenta com a presença de auditores independentes. A relação esperada era contrária, podendo-se concluir que os mecanismos de governança podem não ser eficientes no controle de TPRs. A influência positiva dos auditores independentes no valor das transações pode estar associada ao fato de que as variáveis de governança têm impacto limitado nos preços das TPRs com acionistas controladores (Cheung, Qi, Raghavendra Rau, \& Stouraitis, 2009).

Os resultados não significativos referentes à governança corporativa se assemelham a estudos anteriores realizados no Brasil e refutam a hipótese 4 do trabalho. Oda (2011) analisou as TPRs e o valor de mercado das companhias pertencentes ao Novo Mercado. A autora não inferiu relação entre os mecanismos de governança corporativa e o resultado de TPRs. Souza et al. (2013) também reportaram que não há evidências de que o valor das TPRs possa ter alguma relação com a adoção de níveis diferenciados de governança corporativa no Brasil.

Outras pesquisas, também realizadas no país (Silveira et al., 2008; Souza \& Bortolon, 2014), identificaram relações negativas entre o nível de utilização de TPRs e a qualidade da governança corporativa, sendo que a adoção de mecanismos eficazes de governança corporativa poderá minimizar o impacto das TPRs no valor da empresa. Em função dessas divergências, a governança corporativa é uma temática que deve ser novamente explorada por outros estudos que tenham como objetivo compreender os mecanismos das TPRs.

Tabela 4

\section{Fatores explicativos das TPRs com controladoras/controladas}

\begin{tabular}{|c|c|c|c|c|c|}
\hline \multirow[b]{2}{*}{ Variáveis } & \multicolumn{5}{|c|}{ Log do Valor Total de TPRs com Controladoras/Controladas } \\
\hline & $\begin{array}{c}\text { Quantil } \\
0,10\end{array}$ & $\begin{array}{c}\text { Quantil } \\
0,25\end{array}$ & $\begin{array}{c}\text { Quantil } \\
0,50\end{array}$ & $\begin{array}{c}\text { Quantil } \\
0,75\end{array}$ & $\begin{array}{c}\text { Quantil } \\
0,90\end{array}$ \\
\hline LD & 0,0113 & 0,0122 & $0,0133 * *$ & $0,0142 *$ & 0,0149 \\
\hline $\mathrm{ROA}$ & 0,1433 & 0,0533 & $-0,0583$ & $-0,1506$ & $-0,2259$ \\
\hline Qtobin & 0,0018 & $-0,0032$ & $-0,0094$ & $-0,0145$ & $-0,0187$ \\
\hline $\mathrm{LRCL}$ & 0,0153 & 0,0148 & 0,0143 & 0,0138 & 0,0134 \\
\hline Alav & $-0,1413$ & $-0,1494$ & $-0,1594 * *$ & $-0,1677^{*}$ & $-0,1745$ \\
\hline Tang & 0,1148 & 0,0556 & $-0,0179$ & $-0,0787$ & $-0,1283$ \\
\hline Audit & 0,1758 & $0,1970 * *$ & $0,2234 * \star *$ & $0,2451 * * *$ & $0,2629 * * *$ \\
\hline Big4 & 0,0525 & 0,0322 & 0,0070 & $-0,0137$ & $-0,0307$ \\
\hline NM & 0,0453 & 0,0229 & $-0,0048$ & $-0,0278$ & $-0,0465$ \\
\hline Estrang & $0,1776 *$ & $0,1834 * \star \star$ & $0,1905 * * *$ & $0,1964 * \star \star$ & $0,2013 * * *$ \\
\hline EF Ano & Sim & Sim & Sim & Sim & Sim \\
\hline Nºbs. & 261 & 261 & 261 & 261 & 261 \\
\hline
\end{tabular}

$(* * *),(* *),(*)$ estatisticamente significante a $1 \%, 5 \%$ e $10 \%$, respectivamente.

Legenda: nessa tabela, apresentam-se os resultados do modelo de regressão quantílica com painel fixo, considerando operações com controladoras/controladas, sendo LD: logaritmo do desvio de direito; ROA: rentabilidade do ativo para mensurar o desempenho; QTobin: Q de Tobin para mensurar o valor da empresa; LRECL: logaritmo da receita líquida para mensurar o tamanho; Alav: alavancagem mensurada pelo ativo total; Tang: tangibilidade; Audit: dummy para presença de membros independentes no comitê de auditoria; Big4: dummy para empresas auditadas pelas 4 grandes empresas de auditoria; NM: dummy para presença no novo mercado; Estrang: dummy para presença de capital estrangeiro na estrutura de controle e estrutura de propriedade. Nota: O valor médio do fator de inflação da variância (VIF) para esse modelo é de 1,21.

Fonte: dados da pesquisa. 
Outra variável estatisticamente significativa nesse modelo foi a presença de acionistas estrangeiros. Obteve-se significância em todos os quantis, de forma crescente e positivamente relacionada às TPRs, indicando que a presença desses acionistas, na estrutura de propriedade e controle das empresas piramidais, pode ser uma das formas de incentivo à utilização de TPRs (Cheung, Jing, et al., 2009).

Esse mesmo modelo foi estimado, na Tabela 5, para as TPRs com coligadas, a fim de compreender os fatores explicativos das TPRs para essas empresas. Tem-se um menor volume de transações com essas partes relacionadas, visto que há participação acionária significativa, mas não há controle. Nos resultados, ficou evidenciado que a estrutura indireta das empresas coligadas não é um fator explicativo para as TPRs, pois, apesar de encontrar uma relação negativa entre as variáveis, essa não foi significativa para nenhum quantil.

Tabela 5

Fatores explicativos das TPRs com coligadas

\begin{tabular}{cccccc}
\hline & \multicolumn{5}{c}{ Log do Valor Total de TPRs com Coligadas } \\
\cline { 2 - 6 } Variáveis & $\begin{array}{c}\text { Quantil } \\
\mathbf{0 , 1 0}\end{array}$ & $\begin{array}{c}\text { Quantil } \\
\mathbf{0 , 2 5}\end{array}$ & $\begin{array}{c}\text { Quantil } \\
\mathbf{0 , 5 0}\end{array}$ & $\begin{array}{c}\text { Quantil } \\
\mathbf{0 , 7 5}\end{array}$ & $\begin{array}{c}\text { Quantil } \\
\mathbf{0 , 9 0}\end{array}$ \\
\hline LD & $-0,0096$ & $-0,0068$ & $-0,0047$ & $-0,0027$ & $-0,0011$ \\
\hline ROA & $-0,0224$ & $-0,0064$ & 0,0055 & $\mathbf{0 , 0 1 6 9 * *}$ & $\mathbf{0 , 0 2 6 1 * *}$ \\
\hline Qtobin & 0,0181 & $-0,0322$ & $-0,0698$ & $-0,1057$ & $-0,1349$ \\
\hline LRCL & 0,0169 & 0,0181 & 0,0189 & 0,0198 & 0,0205 \\
\hline Alav & $-0,1057$ & $-0,0614$ & $-0,0284$ & 0,0032 & 0,0289 \\
\hline Tang & $-\mathbf{0 , 1 3 5 2 * *}$ & $\mathbf{- 0 , 1 2 4 2 * *}$ & $-\mathbf{0 , 1 1 5 9 * *}$ & $\mathbf{- 0 , 1 0 8 0 * * *}$ & $-\mathbf{0 , 1 0 1 7 * * *}$ \\
\hline Audit & 0,5006 & 0,3834 & 0,2959 & 0,2122 & 0,1443 \\
\hline Big4 & $-0,5298$ & $\mathbf{- 0 , 4 1 4 1 *}$ & $-\mathbf{0 , 3 2 7 7 * *}$ & $\mathbf{- 0 , 2 4 5 2 * *}$ & $-0,1782$ \\
\hline NM & $-0,1921$ & $-0,0382$ & 0,0767 & 0,1866 & 0,2758 \\
\hline Estrang & 0,2665 & 0,2222 & 0,1891 & 0,1574 & 0,1317 \\
\hline EF Ano & Sim & Sim & Sim & Sim & Sim \\
\hline No Obs. & 78 & 78 & 78 & 78 & 78 \\
\hline
\end{tabular}

$(* * *),\left({ }^{* *}\right),(*)$ estatisticamente significante a $1 \%, 5 \%$ e $10 \%$, respectivamente.

Legenda: nessa tabela, apresentam-se os resultados do modelo de regressão quantílica com painel fixo considerando operações com controladoras/controladas, sendo LD: logaritmo do desvio de direito; ROA: rentabilidade do ativo para mensurar o desempenho; Q Tobin: Q de Tobin para mensurar o valor da empresa; LRECL: logaritmo da receita líquida para mensurar o tamanho; Alav: alavancagem mensurada pelo ativo total; Tang: tangibilidade; Audit: dummy para presença de membros independentes no comitê de auditoria; Big4: dummy para empresas auditadas pelas 4 grandes empresas de auditoria; NM: dummy para presença no novo mercado; Estrang: dummy para presença de capital estrangeiro na estrutura de controle e estrutura de propriedade. Nota: O valor médio do fator de inflação da variância (VIF) para esse modelo é de 1,26.

Fonte: Dados da pesquisa. 
O referido resultado rejeita parcialmente a hipótese 1 e pode estar relacionado com o fato de que as coligadas possuem participação acionária significativa em outras empresas, mas não as controlam. Assim, pode não haver interesse dos controladores em realizar TPRs entre esses tipos de empresas. Infere-se que esse resultado corrobora com o exposto por Souza et al. (2013), os quais identificaram um número mais expressivo de empresas controladoras/controladas relacionadas a TPRs em comparação com as empresas coligadas. De acordo com os autores, isso atesta que as empresas que adotam TPRs preferem a relação de poder (controle) ao realizar investimentos em outras sociedades.

Como variáveis explicativas dessas operações, têm-se o ROA, a tangibilidade e a auditoria por Big Four. Nesse caso, a rentabilidade do ativo possui coeficientes positivos e significativos nos quantis $75 \% \mathrm{e}$ 90\%. Seu efeito no valor das TPRs é pequeno (cerca de $0,01 \%$ ), demonstrando que o maior desempenho contribui positivamente para o aumento do valor transacionado, refutando a hipótese 3 do trabalho. A significância estatística nos quantis superiores reporta que o ROA é um fator explicativo para as TPRs com coligadas quando alcançar determinado nível.

De forma geral, a relação entre TPRs e desempenho tem demonstrado que há um efeito negativo dessas operações (Wang et al., 2019), já que as companhias poderão ter incentivos para ganhos oportunistas (Kang et al., 2014), reduzindo seu desempenho. No entanto, sob a ótica das transações eficientes, as TPRs poderão reduzir custos e resultar em crescimento financeiro (Maheshwari \& Gupta, 2018; Wang et al., 2019; Wong et al., 2015), o que justifica os achados nesta pesquisa.

Sobre a tangibilidade, há uma influência negativa presente em todos os quantis, com coeficientes variando de $0,10 \%$ a $0,13 \%$. Esse efeito mostra que a presença de ativos imobilizados reduz o volume transacionado entre empresas coligadas, corroborando com Souza e Bortolon (2014).

$\mathrm{O}$ último fator explicativo das TPRs com coligadas corresponde à auditoria pelas quatro grandes empresas do setor (Big4). Como esperado pela literatura (Kang et al., 2014), a governança corporativa, a partir da variável mencionada, reduz o volume de TPRs com coligadas. O resultado corrobora com a hipótese 4 do trabalho. A significância está em três quantis, com coeficientes oscilando entre 0,24 e 0,41.

Nessa perspectiva, observa-se que algumas variáveis não foram significativas. Comparativamente, em ambos os modelos, não houve significância do valor da empresa, representado pelo Q de Tobin, do tamanho e da adesão ao Novo Mercado. Sobre o valor da firma, a tendência na literatura é de uma associação negativa com as TPRs, em função de possíveis interesses oportunistas por parte dos controladores (Bona-Sánchez et al., 2017; Cheung, Jing, et al., 2009). Quanto ao tamanho, esperava-se uma influência positiva, pela possibilidade de níveis mais elevados de TPRs em empresas maiores. Cabe salientar que as associações relatadas nessas duas variáveis geralmente acontecem em modelos gerais, ou seja, aqueles que não separam as TPRs entre controladoras/controladas e coligadas.

Com relação à governança corporativa, algumas medidas foram significativas nos modelos e em alguns quantis. Nesse sentido, sabendo que os acionistas controladores poderão utilizar as TPRs como forma de alavancar seu poder de voto, torna-se relevante compreender de que forma e quais as medidas de governança corporativa poderão reduzir a utilização dessas fontes de benefícios privados. Por tudo isso, as variáveis não significativas nos modelos podem ser utilizadas em estudos futuros.

Sob o arcabouço teórico da teoria da Agência, havendo interesses distintos entre as partes, poderão ocorrer conflitos entre o detentor de capital e o administrador. Nessa mesma linha, países que possuem fraca proteção legal contra expropriação acumulam outro conflito de interesses, gerado por acionistas majoritários e minoritários. Portanto, os controladores poderão utilizar mecanismos que mantenham/ aumentem seu poder de voto, situação que poderá reduzir o valor da empresa e expropriar acionistas minoritários. Entre esses mecanismos, adotou-se, no estudo, a utilização de transações com partes relacionadas e a propriedade piramidal. As evidências encontradas suportam a relação entre essas temáticas, demonstrando que as empresas poderão realizar TPRs ao longo da cadeia de propriedades da pirâmide e, com isso, manter o controle com o menor nível de investimentos, gerando desvios de direitos. Na sequência, são apresentadas as conclusões do trabalho. 


\section{Conclusões}

A presente pesquisa teve como objetivo analisar os fatores explicativos das TPRs, em empresas controladoras/controladas e coligadas com estrutura piramidal no Brasil. Realizou-se um estudo longitudinal, no período de 2010 a 2017, em 153 empresas com estrutura piramidal, nas quais foram estimadas regressões quantílicas para identificar os fatores explicativos. De forma geral, conclui-se que a estrutura piramidal é um fator explicativo para as TPRs nas operações entre controladoras/controladas, não rejeitando a hipótese 1. Diferentemente, para empresas coligadas, não houve influência do desvio de direito no valor total das TPRs. Pode-se relacionar tal fato com o volume transacionado entre essas partes, já que existem mais operações com controladoras/controladas do que com coligadas. Silveira et al. (2008) mostraram que operações com controladoras/controladas correspondem a 75,6\% do total, enquanto que para coligadas a apenas $1,4 \%$. Com isso, a existência de subordinação entre controladoras/controladas poderá estimular o uso de TPRs para interesses específicos e aumento no nível de desvios.

Quanto à análise do desempenho contábil, as evidências empíricas revelaram que este é um fator explicativo positivo das TPRs com coligadas. Por haver poucas operações com essas partes, as TPRs podem não ter o poder de destruir o valor dessas firmas. Já, com relação ao Q de Tobin, esse não foi significativo para nenhuma análise, sendo que tal resultado pode estar associado ao nível de desvio dessas empresas. Para Kang et al. (2014), a destruição de valor da firma por TPRs só ocorre quando os conflitos de interesses entre acionistas controladores e minoritários são severos.

No que se refere à influência da governança corporativa nas TPRs, observou-se que houve papel de moderação apenas para transações com coligadas. No modelo de controladoras/controladas, o sinal esperado foi contrário, demonstrando que a governança corporativa não reduz o valor transacionado entre essas empresas. Evidências anteriores também demonstraram que os mecanismos de governança têm pouco impacto sobre as TPRs, sendo seus resultados ainda inconclusivos (Cheung, Qi, et al., 2009; Oda, 2011).

A contribuição teórica do estudo das transações com partes relacionadas se dá por ser uma temática pouco explorada no contexto nacional, especialmente, ao considerar as particularidades das relações, como as operações com controladoras/controladas e coligadas. No Brasil, as razões para a formação de estruturas piramidais são controversas, necessitando de evidências empíricas, visto a carência de literatura sobre tal temática (Aldrighi \& Postali, 2011).

A contribuição empírica consiste na aplicação do método de regressão quantílica para dados em painel, que é inovador e apropriado para análises longitudinais. Com isso, os resultados representam uma contribuição para a literatura brasileira, demonstrando como a influência da estrutura piramidal se dá em cada um dos níveis de valores de TPRs executadas entre controladoras/controladas e coligadas.

Os resultados podem ser úteis para melhoria dos processos de divulgação de informações atinentes às empresas listadas em bolsa. Por exemplo, muitas descrições de TPRs são gerais, não demonstrando de forma clara a operação que foi realizada. Assim, em função da recente obrigatoriedade de divulgação de TPRs (2010), pode-se buscar padronização e melhoria na divulgação de informações, sendo resultados úteis para as instituições que regulamentam o mercado de capitais.

Além disso, a não moderação da governança corporativa pode sinalizar que o ambiente legal do mercado brasileiro permite que os acionistas controladores utilizem estratégias para garantir seu poder de voto. Em função disso, os resultados interessam aos acionistas minoritários, que poderão atentar-se à realização de TPRs entre empresas que pertencem à estrutura piramidal, visto o risco de expropriação e benefícios privados. Em suma, considerando a amplitude de temas como valor da firma, desempenho e governança corporativa, evidências do cenário brasileiro são importantes, particularmente, ao considerar as TPRs e a propriedade piramidal.

As limitações gerais são inerentes ao método de pesquisa empregado, o qual está suscetível à endogeneidade presente nos estudos de governança corporativa. Outra limitação intrínseca ao trabalho é a própria definição das variáveis, como, por exemplo, a utilização de variáveis binárias para alguns aspectos do trabalho. 
Quanto ao recorte temporal, a limitação está no último ano analisado (2017), visto que, até o momento da coleta de dados, eram os resultados que estavam disponíveis na fonte de informação. Já o ano inicial é justificado pelo começo da divulgação desses dados no formulário de referência. Tem-se ainda que, devido ao foco do estudo, utilizou-se o critério de seleção das estruturas piramidais baseado na existência de pelo menos um intermediário listado. Essa definição foi utilizada como forma de analisar se o acesso desse intermediário à bolsa de valores poderia ter relevância na execução de TPRs.

Como sugestões para pesquisas futuras, poderão ser realizados trabalhos mais descritivos a respeito das TPRs. Devido à quantidade de informações qualitativas, seria apropriado separar as transações por sua natureza e verificar seus determinantes. As TPRs também podem ser analisadas sob outros aspectos, visto que tais operações são usadas como um canal de expropriação de acionistas minoritários. São encontradas literaturas sobre a interação entre TPRs, desempenho e valor da firma, mas os resultados empíricos para o Brasil ainda são escassos, especialmente, no mesmo recorte temporal dessa pesquisa. Basicamente, as evidências servem como base norteadora para a formulação de novas hipóteses de pesquisa, as quais possam contribuir para uma melhor compreensão das motivações para a formação de estruturas piramidais e realização de TPRs no mercado brasileiro.

\section{Referências}

Aldrighi, D. M. (2014). Concentração da Propriedade do Capital e Controle das Empresas no Brasil. In: XIV Encontro Brasileiro de Finanças.

Aldrighi, D. M., \& Mazzer Neto, R. (2005). Estrutura de Propriedade e de Controle das Empresas de Capital Aberto no Brasil. Revista de Economia Política, 25(98), 115-137.

Aldrighi, D. M., \& Postali, A. F. (2010). Business Groups in Brazil. In A. M. Colpan, T. Hikino, \& J. R. Lincoln (Eds.), The Oxford Handbook of Business Groups (Oxford Uni). Nova York: Oxford University Press. https://doi.org/10.1093/oxfordhb/9780199552863.001.0001

Aldrighi, D. M., \& Postali, A. F. (2011). Propriedade Piramidal das Empresas no Brasil. Revista EconomiA, 12(1), 27-48.

Aldrighi, D. M., Postali, F. A. S., \& Diaz, M. D. M. (2018). Corporate Governance and Pyramidal Ownership: The Role of Novo Mercado. Brazilian Review of Finance, 16(1), 5-38.

Almeida, M. R. de. (1987). Sociedades coligadas, controladas e controladoras (holding). Revista de Ciência Política; v. 30, n. 2 (1987). Retrieved from http://bibliotecadigital.fgv.br/ojs/index.php/rcp/article/ view/60140/58457

Almeida, H., Kim, C. S., \& Kim, H. B. (2015). Internal Capital Markets in Business Groups: Evidence from the Asian Financial Crisis. Journal of Finance, 70(6), 2539-2586. https://doi.org/10.1111/jofi.12309

Almeida, H., Park, S. Y., Subrahmanyam, M. G., \& Wolfenzon, D. (2011). The structure and formation of business groups: Evidence from Korean chaebols. Journal of Financial Economics, 99(2), 447-475. https://doi.org/10.1016/j.jfineco.2010.08.017

Almeida, H., \& Wolfenzon, D. (2006). A Theory of Pyramidal Ownership. The Journal of Finance, 56(6), 2637-2680. https://doi.org/10.1111/j.1540-6261.2006.01001.x

Bebchuk, L. A., Kraakman, R., \& Triantis, G. G. (2000). Ownership and Dual Class Equity: The Mechanisms and Agency Costs of Separating Control from Cash-Flow Rights. In Concentrated Corporate Ownership (pp. 445-460). University of Chicago Press.

Bena, J., \& Ortiz-Molina, H. (2013). Pyramidal ownership and the creation of new firms. Journal of Financial Economics, 108(3), 798-821. https://doi.org/10.1016/j.jfineco.2013.01.009 
Bertrand, M., Mehta, P., \& Mullainathan, S. (2002). Ferreting Out Tunneling : An Application to Indian Business. The Quarterly Journal of Economics, 117(1), 121-148.

Bertrand, M., \& Mullainathan, S. (2003). Pyramids. Journal of the European Economic Association, $1(2-3), 478-473$.

Bhuiyan, M. B. U., \& Roudaki, J. (2018). Related party transactions and finance company failure: New Zealand evidence. Pacific Accounting Review, 30(2), 199-221. https://doi.org/10.1108/PAR-11-2016-0098

Bona-Sánchez, C., Fernández-Senra, C. L., \& Pérez-Alemán, J. (2017). Related-party transactions, dominant owners and firm value. BRQ Business Research Quarterly, 20(1), 4-17. https://doi. org/10.1016/j.brq.2016.07.002

Bortolon, P. M. (2010). Determinantes e consequências das decisões de estrutura de propriedade: estrutura piramidal e unificação de ações. Universidade Federal do Rio de Janeiro. Tese.

Cai, W., Zeng, C. C., Lee, E., \& Ozkan, N. (2016). Do business groups affect corporate cash holdings? Evidence from a transition economy. China Journal of Accounting Research, 9(1), 1-24. https://doi. org/10.1016/j.cjar.2015.10.002

Carvalhal da Silva, A. L. (2005). Governança Corporativa e Decisóes Financeiras no Brasil (Mauad). Rio de Janeiro.

Chen, W., Li, S., \& Chen, C. X. (2017). How much control causes tunneling? Evidence from China. China Journal of Accounting Research, 10(3), 231-245. https://doi.org/10.1016/j.cjar.2016.10.001

Cheung, Y. L., Jing, L., Lu, T., Rau, P. R., \& Stouraitis, A. (2009). Tunneling and propping up: An analysis of related party transactions by Chinese listed companies. Pacific Basin Finance Journal, 17(3), 372393. https://doi.org/10.1016/j.pacfin.2008.10.001

Cheung, Y. L., Qi, Y., Raghavendra Rau, P., \& Stouraitis, A. (2009). Buy high, sell low: How listed firms price asset transfers in related party transactions. Journal of Banking and Finance, 33(5), 914-924. https://doi.org/10.1016/j.jbankfin.2008.10.002

Cho, S., \& Lim, K. M. (2018). Tunneling by Related-party Transactions: Evidence from Korean Conglomerates. Asian Economic Journal, 32(2), 147-164. https://doi.org/10.1111/asej.12146

Claessens, S., Djankov, S., \& Lang, L. H. . (2000). The separation of ownership and control in East Asian Corporations. Journal of Financial Economics, 58(1-2), 81-112. https://doi.org/10.1016/ S0304-405X(00)00067-2

CVM. (2010). Aprova o pronunciamento técnico CPC 05 (R1) do comitê de pronunciamentos contábeis, que trata das divulgações sobre partes relacionadas. Rio de Janeiro.

Da Silva, Á. F., \& Neves, P. (2018). Portugal: changing environment and flexible adaptation. In Business Groups in the West: Origins, Evolution, and Resilience (p. 592). Oxford University Press.

Faccio, M., \& Lang, L. H. P. (2002). The ultimate ownership of Western European corporations. Journal of Financial Economics, 65, 365-395. Retrieved from http://citeseerx.ist.psu.edu/viewdoc/download ?doi=10.1.1.520.9523\&rep=rep1\&type $=$ pdf

Fang, J., Pittman, J., Zhang, Y., \& Zhao, Y. (2017). Auditor Choice and Its Implications for Group-Affiliated Firms. Contemporary Accounting Research, 34(1), 39-82. https://doi.org/10.1111/1911-3846.12276

Firth, M., Fung, P. M. Y., \& Rui, O. M. (2007). Ownership, two-tier board structure, and the informativeness of earnings - Evidence from China. Journal of Accounting and Public Policy, 26(4), 463-496. https:// doi.org/10.1016/j.jaccpubpol.2007.05.004

Gordon, E. A., Henry, E., \& Palia, D. (2004). Related Party Transactions and Corporate Governance. Advances in Financial Economics, 9, 1-27. https://doi.org/10.1016/S1569-3732(04)09001-2 
Granovetter, M. (2005). Business Groups and Social Organization. In The handbook of economic sociology (pp. 3-25).

Greene, W. H. (2012). Econometric Analysis (Vol. 2). New York: Pearson.

Hansen, L. P. (1982). Large Sample Properties of Generalized Method of Moments Estimators. Econometrica, 50(4), 1029-1054. https://doi.org/10.2307/1912775

Himmelberg, C. P., Hubbard, G., \& Palia, D. (1999). Understanding the determinants of managerial ownership and the link between ownership and performance. Journal of Financial Economics, 53, 353-384. https://doi.org/10.1016/S0304-405X(01)00085-X

Huyghebaert, N., \& Wang, L. (2012). Expropriation of Minority Investors in Chinese Listed Firms: The Role of Internal and External Corporate Governance Mechanisms. Corporate Governance: An International Review, 20(3), 308-332. https://doi.org/10.1111/j.1467-8683.2012.00909.x

Jian, M., \& Wong, T. J. (2010). Propping through related party transactions. Review of Accounting Studies, 15(1), 70-105. https://doi.org/10.1007/s11142-008-9081-4

Johnson, S., La Porta, R., Lopez-de-Silanes, F., \& Shleifer, A. (2000). Tunneling. American Economic Review, 90(2), 22-27. https://doi.org/10.1257/aer.90.2.22

Kang, M., Lee, H. Y., Lee, M. G., \& Park, J. C. (2014). The association between related-party transactions and control-ownership wedge: Evidence from Korea. Pacific Basin Finance Journal, 29, 272-296. https://doi.org/10.1016/j.pacfin.2014.04.006

Kim, S. H., \& An, Y. (2018). The effect of ownership-control disparity on the Chinese firm's real activity earnings management. Pacific Accounting Review, 30(4), 482-499. https://doi.org/10.1108/PAR01-2018-0003

Kirch, G., Procianoy, J. L., \& Terra, P. R. S. (2014). Restrições financeiras e a decisão de investimento das firmas brasileiras. Revista Brasileira de Economia, 68(1), 103-123. https://doi.org/10.1590/s003471402014000100006

La Porta, R., Lopez-de-silanes, F., \& Shleifer, A. (1999). Corporate Ownership around the World Corporate Ownership Around the World. Journal of Finance, 54(2), 471-517.

La Porta, R., Lopez-de-silanes, F., Shleifer, A., \& Vishny, R. (2000). Investor protection and corporate governance. Journal of Financial Economics, 58, 3-27. https://doi.org/10.1016/S0304405X(00)00065-9

Lei, A. C. H., \& Song, F. M. (2011). Connected transactions and firm value: Evidence from Chinaaffiliated companies. Pacific Basin Finance Journal, 19(5), 470-490. https://doi.org/10.1016/j. pacfin.2011.07.002

Machado, J. A. F., \& Santos Silva, J. M. C. (2019). Quantiles via moments. Journal of Econometrics, 213(1), 145-173. https://doi.org/10.1016/j.jeconom.2019.04.009

Maheshwari, Y., \& Gupta, P. (2018). Ownership structures and effects of related lending and loan guarantees on firm performance in business groups. Asian Journal of Accounting and Governance, 9, 77-86. https://doi.org/10.17576/ajag-2018-09-07

Matos, O. M. da S. P., \& Galdi, F. C. (2014). O Impacto Das Transações Com Partes Relacionadas Na Performance Operacional Das Companhias Listadas Na Bm\&Fbovespa. Contabilidade Vista \& Revista, 25(2), 84-97.

Oda, P. (2011). Transações com partes relacionadas, governança corporativa e desempenho: um estudo com dados em painel. Universidade de São Paulo. Dissertação.

Perlin, M. S., Kirch, G., \& Vancin, D. (2019). Accessing financial reports and corporate events with GetDFPData. Revista Brasileira de Finanças, 17(3), 85-108. 
Rahmat, M. M., Mohd Amin, H. A., \& Mohd Saleh, N. (2018). Controlling Shareholders' Proxy and Multiple Directorships: Insight Evidence from Related Party Transactions. Asian Journal of Accounting and Governance, 9, 27-40. https://doi.org/10.17576/ajag-2018-09-03

Ramsey, J. B. (1969). Tests for Specification Errors in Classical Linear Least-Squares Regression Analysis. Journal of the Royal Statistical Society: Series B (Methodological), 31(2), 350-371. https://doi. org/10.1111/j.2517-6161.1969.tb00796.x

Silveira, A. D. M. da, Prado, V. M., \& Sasso, R. (2008). Transações com partes relacionadas: estratégias jurídicas e relação com a governança corporativa e valor das empresas no Brasil. Estudos Em Governança Corporativa, 1-73.

Souza, J. A. S., \& Bortolon, P. M. (2014). Transações com partes relacionadas: determinantes e impactos no desempenho das empresas. XIV Encontro Brasileiro de Finanças.

Souza, M. M., Knupp, P. de S., \& Borba, J. A. (2013). Características Associadas às Transações com Partes Relacionadas nas Empresas de Capital Aberto Brasileiras. Contabilidade, Gestão e Governança, 16(3), 124-137.

Tirole, J. (2006). The Theory of Corporate Finance (Princeton, Vol. 67). New Jersey.

Wang, H. Da, Cho, C. C., \& Lin, C. J. (2019). Related party transactions, business relatedness, and firm performance. Journal of Business Research, 101(January), 411-425. https://doi.org/10.1016/j. jbusres.2019.01.066

Wong, R. M. K., Kim, J. B., \& Lo, A. W. Y. (2015). Are related-party sales value-adding or value-destroying? Evidence from China. Journal of International Financial Management and Accounting, 26(1), 1-38. https://doi.org/10.1111/jifm.12023

Yeh, Y. H., Shu, P. G., Lee, T. S., \& Su, Y. H. (2009). Non-tradable share reform and corporate governance in the chinese stock market. Corporate Governance: An International Review, 17(4), 457-475. https:// doi.org/10.1111/j.1467-8683.2009.00754.x 